\title{
Raman selection rules in uniaxial media: The nonpolar modes of $\mathrm{MnGa}_{2} \mathrm{Se}_{4}$
}

\author{
P. Alonso-Gutiérrez, M. L. Sanjuán,* and M. C. Morón \\ Instituto de Ciencia de Materiales de Aragón (Universidad de Zaragoza-Consejo Superior de Investigaciones Científicas), \\ Facultad de Ciencias, 50009 Zaragoza; Spain \\ (Received 27 January 2004; published 22 February 2005)
}

\begin{abstract}
It is known that optically anisotropic media can change the polarization of light propagating inside them. As regards Raman spectroscopy, this affects the light intensity measured in different geometrical configurations and results in an apparent unfulfillment of selection rules. We present an experimental and theoretical study of such effects in the defect chalcopyrite semiconductor $\mathrm{MnGa}_{2} \mathrm{Se}_{4}$. Optical anisotropy is taken into account by including in the calculation of Raman intensities the phase difference appearing between ordinary and extraordinary waves as they propagate, in uniaxial media, with different velocities. Birefringence can be obtained from Raman measurements provided that the distance run by the light is known.
\end{abstract}

DOI: 10.1103/PhysRevB.71.085205

PACS number(s): 78.30.-j, 42.70.Mp, 63.20.-e, 75.50.Pp

\section{INTRODUCTION}

$\mathrm{MnGa}_{2} \mathrm{Se}_{4}$ is a ternary, ordered-vacancy semiconductor with a defect-chalcopyrite structure (space group $I \overline{4})^{1}$ and potential technological applications in the field of (linear and nonlinear) optical and optoelectronic applications. ${ }^{2-5}$ In this particular compound, the presence of Mn ions adds a magnetic behavior that opens the way to magneto-optical properties. In view of its technological interest, it would be convenient to have a complete characterization of its optical and electronic properties. Surprisingly, reports on refraction indexes and/or birefringence of ordered-vacancy compounds are scarce. ${ }^{4,6-9}$ They are not available for $\mathrm{MnGa}_{2} \mathrm{Se}_{4}$.

From the structural point of view, the properties of $\mathrm{MnGa}_{2} \mathrm{Se}_{4}$ are dominated, by comparison with its parental zinc-blende compounds, by the presence of cation asymmetry and ordered vacancies, yielding a tetragonal structure with $c \approx 2 a[a=5.677(1) \AA, c=10.761(2) \AA] .{ }^{10}$ In turn, cell doubling leads to optical anisotropy and behavior as a uniaxial medium. As opposed to cubic zinc-blende compounds and depending on the value of the birefringence, this opens the possibility of reaching phase-matching conditions. By analogy with related compounds, such as chalcopyrites $^{5,11}$ and other ordered-vacancy compounds,, ,7-9 a relatively high birefringence is expected for $\mathrm{MnGa}_{2} \mathrm{Se}_{4}$. We are thus in face of a compound that presents magnetic behavior at low temperatures, ${ }^{12}$ optical anisotropy with a probably high birefringence, polar character, and noncentrosymmetric structure. The combination of all these properties also results in a wealth of phenomena, as regards its vibrational properties, that deserves a close spectroscopic study.

In a previous work ${ }^{13}$ we presented a preliminary study of the Raman spectrum of the $\mathrm{Zn}_{1-x} \mathrm{Mn}_{x} \mathrm{Ga}_{2} \mathrm{Se}_{4}$ system focusing on the evolution of the spectrum across the series in relation with structural disorder. A preliminary assignment of mode symmetry was made for $\mathrm{MnGa}_{2} \mathrm{Se}_{4}$. However, in the course of that work we were aware that a full understanding of the vibrational properties of anisotropic media requires taking into account its anisotropic optical properties. In fact this problem is not restricted to vibrational spectroscopy but affects in general any experiment concerning light propagation in anisotropic media. Its importance, for instance, was made evident in Ref. 14 in relation with two-magnon light scattering in ladder systems. As early as in 1966 these effects were found to be responsible for Raman depolarization in calcite. ${ }^{15}$ In this work we present a theoretical and experimental study of polarization properties in the Raman spectrum of $\mathrm{MnGa}_{2} \mathrm{Se}_{4}$. This compound was selected from the $\mathrm{Zn}_{1-x} \mathrm{Mn}_{x} \mathrm{Ga}_{2} \mathrm{Se}_{4}$ series because it shows minimum structural disorder, since $\mathrm{Mn}$ and Ga cations occupy well-defined crystallographic sites, contrary to the other end compound, $\mathrm{ZnGa}_{2} \mathrm{Se}_{4}$, where $\mathrm{Zn}$ and half of the $\mathrm{Ga}$ cations share the same site. ${ }^{16}$ According to the ordered character of the structure, the spectrum of $\mathrm{MnGa}_{2} \mathrm{Se}_{4}$ shows very narrow peaks with clear polarization properties, which indicates good crystallinity.

\section{EXPERIMENTAL DETAILS}

Single crystals of $\mathrm{MnGa}_{2} \mathrm{Se}_{4}$ were grown by the chemical vapor transport method in the presence of iodine as the transport agent. ${ }^{12,17}$ Raman experiments were performed at room temperature in a Dilor $X Y$ spectrometer with a diode array detector. Light from an $\mathrm{Ar}^{+}$laser was focused onto the sample through a $\times 50$ microscope objective lens. For a specific experiment a dye laser at $568.4 \mathrm{~nm}$ was used. The power at the sample was $\simeq 1 \mathrm{~mW}$ and the spectral resolution better than $3 \mathrm{~cm}^{-1}$.

\section{EXPERIMENTAL RESULTS AND INTERPRETATION}

Available single crystals of $\mathrm{MnGa}_{2} \mathrm{Se}_{4}$ are too small (about $2 \mathrm{~mm}$ maximum side) to be cut along selected planes. Therefore, only natural growth planes could be used, the most frequent one being the tetragonal (112) face [(111) in pseudocubic axes. For convenience we shall use cubic notation in the following]. Fortunately, one of the samples tested was found to present (001) and quasi (110) faces, the latter being finally identified as a $\left(11 \frac{1}{2}\right)$ pseudocubic face. We have thus performed measurements in three planes: (001), (111), and $\left(11 \frac{1}{2}\right)$, where the angle $\theta$ formed by the plane normal and the optic axis is $0,55.74$ and 70.53 degrees, respectively (see 


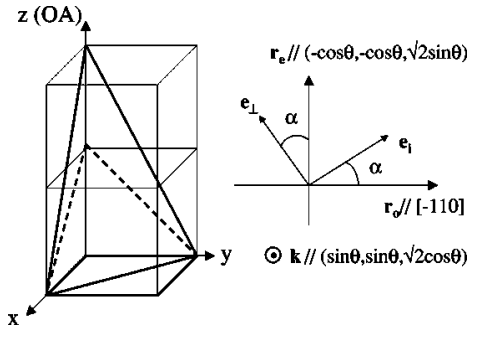

FIG. 1. Schematic diagram of the unit cell of $\mathrm{MnGa}_{2} \mathrm{Se}_{4}$ showing the three crystallographic planes (left) and reference system (right) used in this work. $\theta$ is the angle formed by the plane normal and the optic axis $z$.

Fig. 1). Raman measurements were performed onto these planes in backscattering geometry as a function of the angle $\alpha$ formed by the incident electric field and the [110] direction, which is common to all three planes. The sample was rotated about the normal to the surface, which is also the direction of incident and scattered light. At each sample position the analyzer was rotated so as to allow for recording spectra in parallel and in crossed configurations, in which the electric field of the scattered light $\mathbf{E}_{\mathrm{s}}$ is parallel or perpendicular to that of the incident light $\mathbf{E}_{\mathrm{i}}$, respectively. The intensity of each mode is then plotted as a function of sample rotation both in parallel and in crossed configurations. We call such a plot a rotational diagram. The experimental arrangement is schematically depicted in Fig. 1.

According to factor group analysis for the $I \overline{4}$ symmetry of $\mathrm{MnGa}_{2} \mathrm{Se}_{4}, 13$ Raman active modes are expected, which decompose as $3 A+5 B+5 E .{ }^{13} B$ and $E$ modes are polar and thus also IR active. $A$ modes are nonpolar. For the ten polar modes, different $L O$ and $T O$ frequencies are a priori expected. The behavior of polar modes in uniaxial crystals presents a further degree of complexity since, in that case, not only the photons but also the phonons show anisotropic properties, their frequencies and intensities depending on the relative directions of phonon polarization and propagation, and of those with respect to the optic axis. ${ }^{20}$ Since our aim is to see the effect of anisotropy in the polarization properties of Raman modes, in this work we shall restrict to nonpolar $A$ modes, which are identified in $\mathrm{MnGa}_{2} \mathrm{Se}_{4}$ at $\nu \cong 134.7,181.8$ and $207.5 \mathrm{~cm}^{-1} .^{13}$ Polar modes will be the object of a separate paper.

Figure 2 shows the Raman spectrum of $\mathrm{MnGa}_{2} \mathrm{Se}_{4}$ in $z(x x) \bar{z}, z(x y) \bar{z}, z\left(x^{\prime} x^{\prime}\right) \bar{z}$, and $z\left(x^{\prime} y^{\prime}\right) \bar{z}$ configurations on the (001) plane, with $x^{\prime}, y^{\prime}$ at $45^{\circ}$ from $x, y$. The three $A$ modes have been labelled as $A^{1}, A^{2}$, and $A^{3}$. The $A^{3}$ mode is much weaker than the other two, a feature that is also encountered in related compounds, such as $\mathrm{ZnGa}_{2} \mathrm{Se}_{4}$ or $\mathrm{CdGa}_{2} \mathrm{Se}_{4} \cdot{ }^{13,18,19}$ In this plane the three $\mathrm{A}$ modes present, within experimental error, a constant intensity in parallel configuration, their small intensity in crossed configuration (below 5\% of the parallel intensity) being attributable to a polarization leakage.

Figures 3 and 4 show the rotational diagrams of the three $A$ modes in (111) and $\left(11 \frac{1}{2}\right)$ planes, respectively. In all cases $I_{\|}(\alpha)$ presents a minimum and $I_{\perp}(\alpha)$ a maximum at an intermediate angle. We shall first describe the expected intensities

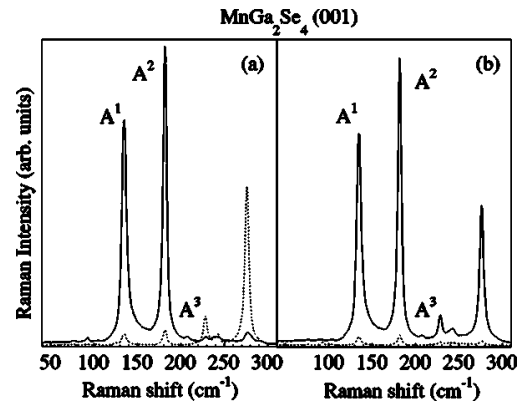

FIG. 2. Raman spectra of $\mathrm{MnGa}_{2} \mathrm{Se}_{4}$ performed onto the (001) plane in four configurations. (a) $z(x x) \bar{z}$ (continuous line) and $z(x y) \bar{z}$ (dashed line). (b) $z\left(x^{\prime} x^{\prime}\right) \bar{z}$ (continuous line) and $z\left(x^{\prime} y^{\prime}\right) \bar{z}$ (dashed line). $\left\{x^{\prime}, y^{\prime}\right\}$ axes are rotated $45^{\circ}$ from $\{x, y\}$.

in the absence of uniaxial effects and will see next why these effects need to be taken into account in order to reproduce the experimental results. For measurements onto a general plane, Raman intensities will be given, except for some factors, by the usual expression

$$
I \propto\left|\sum \mathbf{e}_{\mathbf{i}} R_{i s} \mathbf{e}_{\mathbf{s}}\right|^{2}
$$

where $\mathbf{e}_{\mathbf{i}}, \mathbf{e}_{\mathrm{s}}$ are the polarization vectors of the electric fields of incident and scattered light, respectively, and $R_{i s}$ stands for the Raman tensor pertaining to each allowed mode. ${ }^{20}$ In
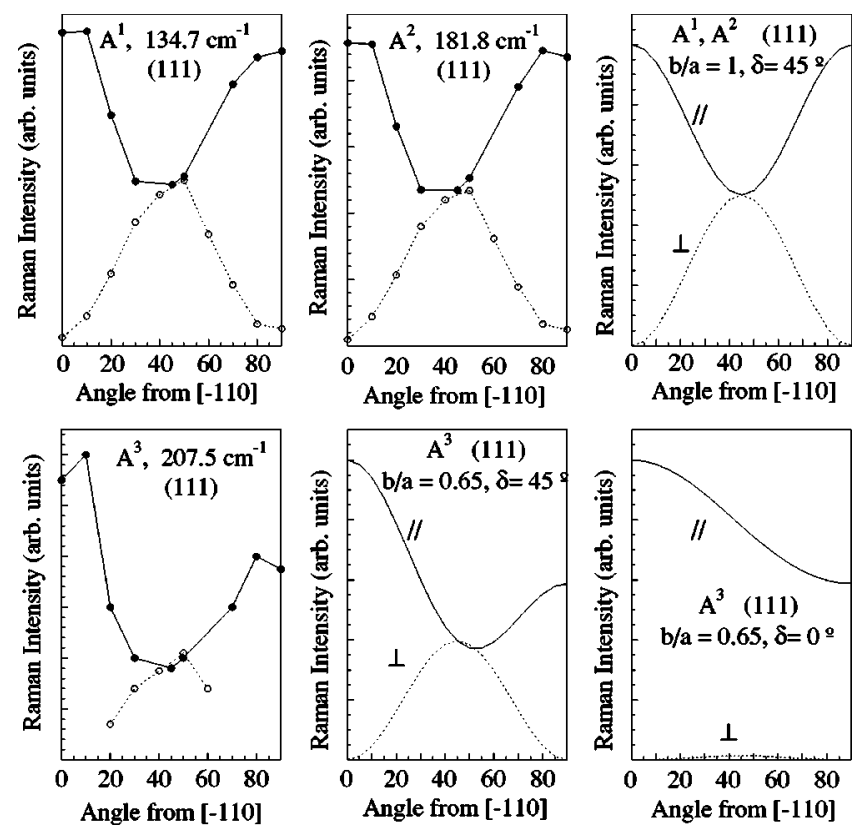

FIG. 3. Rotational diagrams of $A$ modes in (111) plane. (Top) Experimental results for $A^{1}$ and $A^{2}$ modes, and dependency calculated for those modes with inclusion of dephasing effects. If these are not included the calculated intensities (for $a \approx b$ ) are constant and zero, respectively, in parallel and crossed configurations. (Bottom) Experimental result for $A^{3}$ mode, best fit with inclusion of dephasing effects, and expected dependency without such effects for $b \approx 0.65 a$. The intensity scale for the $A^{3}$ mode has been amplified by a factor of more than 20 . 

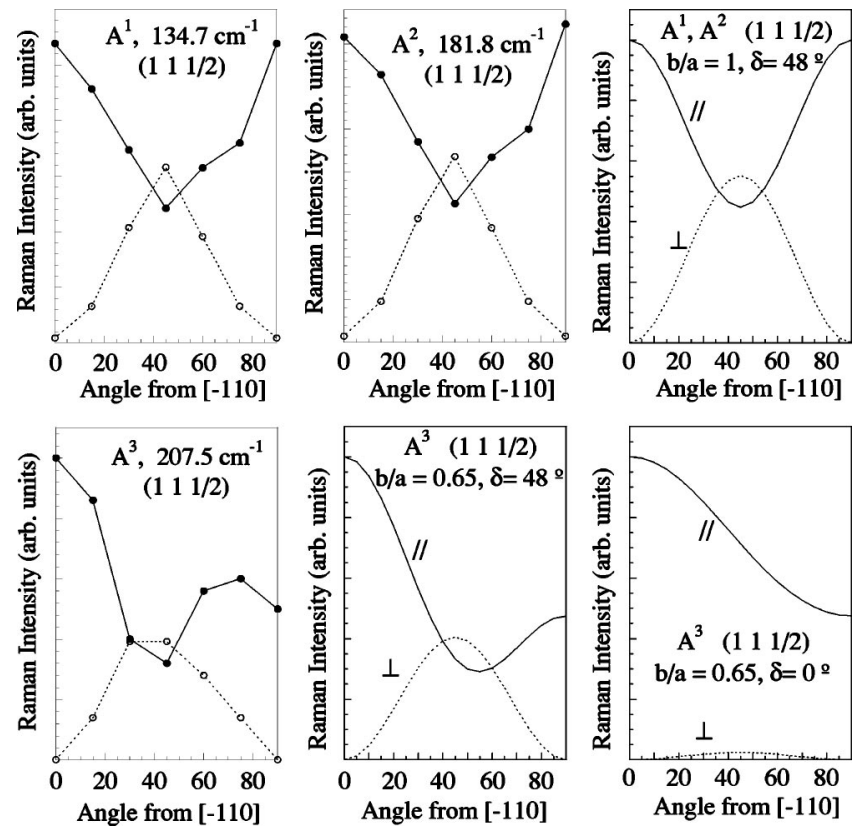

FIG. 4. The same as Fig. 3 but for the $\left(11 \frac{1}{2}\right)$ plane.

our case, the Raman tensor of $A$ modes in $\overline{4}$ point group has the form

$$
\left(\begin{array}{lll}
a & & \\
& a & \\
& & b
\end{array}\right)
$$

For measurements onto the (001) plane only the $[x, y]$ subspace is span and therefore $A$ modes are expected to be isotropic and fully polarized in parallel, i.e., $I_{\|}(001)=a^{2}$, $I_{\perp}(001)=0$ for any direction within that plane. In this plane, the experimental behavior agrees with that expectation. Within the (111) and $\left(11 \frac{1}{2}\right)$ planes we find from Eq. (1)

$$
\begin{aligned}
I_{\|} & =\left(a-(a-b) \sin ^{2} \alpha \sin ^{2} \theta\right)^{2}, \\
I_{\perp} & =(a-b)^{2} \sin ^{2} \alpha \cos ^{2} \alpha \sin ^{4} \theta,
\end{aligned}
$$

where $\alpha$ stands for the angle between the incident electric field and the $[-110]$ direction within each plane. An inconsistency is found when we try to fit the angular dependence of a given mode simultaneously in the two planes. Lets take first the $A^{1}$ and $A^{2}$ modes in the (111) plane. The presence of a minimum in $I_{\|}^{\text {exp }}$ imposes $a / b<0$, in which case $I_{\|}^{c a l}$ goes to zero at an angle $\alpha_{m}$ that depends on $|a / b|$, while $I_{\perp}^{\text {cal }}$ will be symmetrical with maximum at $45^{\circ}$. The fact that $I_{\|}^{\exp }\left(0^{\circ}\right)$ $\approx I_{\|}^{e x p}\left(90^{\circ}\right)$ and the condition $a / b<0$ impose $b=-2 a$, in which case $I_{\|}$should be zero at $45^{\circ}$. Experimentally $I_{\|}\left(45^{\circ}\right)$ is minimum but not zero, and this discrepancy may be due to two reasons: polarization leakage or dephasing effects. Polarization leakage is very small in our measurements and cannot explain intensities $I_{\|}\left(45^{\circ}\right)$ as high as $0.5 I_{\|}\left(0^{\circ}\right)$. On the other hand, dephasing effects could explain the experimental results if it were not for the behavior of these modes in the $\left(11 \frac{1}{2}\right)$ plane. If $b=-2 a$ holds, in the $\left(11 \frac{1}{2}\right)$ plane we would expect $I_{\|}\left(90^{\circ}\right)=(25 / 9) a^{2} \gg I_{\|}\left(0^{\circ}\right)=a^{2}$. On the con- trary, we see that $I_{\|}\left[0^{\circ},(111)\right] \approx I_{\|}\left[90^{\circ},(111)\right]$ $\approx I_{\|}\left[0^{\circ},\left(11 \frac{1}{2}\right)\right] \approx I_{\|}\left[90^{\circ},\left(11 \frac{1}{2}\right)\right]$, which can only be satisfied if $a \approx b$. Regarding their matrix elements, these modes are quasi-isotropic, so that, as a function of $\alpha$, their intensity would be constant in parallel and zero in crossed configuration. Following the same procedure for the $A^{3}$ mode we find $b \approx 0.65 a$. Its expected intensity is given in Figs. 3 and 4 (bottom, right). This behavior is clearly not fulfilled experimentally.

\section{A. Intensities calculated with inclusion of anisotropy effects}

Our experimental results cannot be explained by the usual expression of the Raman intensity (Eq. (1)) without taking into account the depolarization that incident and scattered light suffer as they travel through a uniaxial medium. As described in Ref. 21 , in such a material two waves can propagate in a given direction: the ordinary wave, which propagates with a refraction index $n_{o}$ (ordinary index), and the extraordinary wave, for which there is a dependence of the refraction index $n_{\text {eff }}$ with the direction of propagation, $\mathbf{k}$, of the form

$$
1 / n_{\mathrm{eff}}^{2}=\left(\cos ^{2} \theta\right) / n_{o}^{2}+\left(\sin ^{2} \theta\right) / n_{e}^{2},
$$

$n_{e}$ being the extraordinary index. The polarization of the ordinary wave is always perpendicular to the optic axis and to the direction of light propagation. When $\theta$ is $0^{\circ}$, the polarization of the extraordinary wave is also perpendicular to the optic axis and its refraction index is $n_{o}$. In this case there is no difference between the ordinary and the extraordinary wave. When $\theta=90^{\circ}$, the polarization of the extraordinary wave is parallel to the $c$ axis, and it propagates with a refraction index $n_{e}$. In a general case, the difference between $n_{o}$ and $n_{\text {eff }}$ makes the ordinary and extraordinary waves propagate with different velocity so that a phase difference appears between their electric fields given in radians by

$$
\delta=-\frac{2 \pi}{\lambda}\left(n_{\mathrm{eff}}-n_{o}\right) d,
$$

$\lambda$ being the wavelength of the light, and $d$ the path that the light runs through the medium. We assume that within the medium the propagation is nearly perpendicular to the surface, as in normal incidence. This can be justified by the high refraction index of these materials. We also assume that the dispersion of the extraordinary wave (angle between $\mathbf{D}_{\mathrm{e}}$ and $\mathbf{E}_{\mathrm{e}}$ ) is negligible, so that $\mathbf{E}_{\mathrm{e}}$ is, as $\mathbf{D}_{\mathrm{e}}$, perpendicular to the propagation vector $\mathbf{k}$ and, at the same time, perpendicular to the electric field of the ordinary wave. This can be justified as follows: The dispersion angle $\phi$ is given by ${ }^{22}$

$$
\tan \phi=\frac{\left(n_{e}^{2}-n_{o}^{2}\right) \tan \theta}{n_{e}^{2}+n_{o}^{2} \tan ^{2} \theta} .
$$

If we assume that the birefringence $\left(\Delta=n_{e}-n_{o}\right)$ is small we can make $n_{o}, n_{e} \approx n$ except when subtracted. Then

$$
\tan \phi=\frac{2 n \Delta \tan \theta}{n^{2}\left(1+\tan ^{2} \theta\right)}=\frac{2 \Delta}{n} \sin \theta \cos \theta,
$$

which, for typical values of $\Delta=0.01$ and $n=2.5$, yields $\phi$ $<0.3^{\circ}$. We will also neglect the anisotropy in absorption and 
TABLE I. Parameters of the model ( $\delta$ in degrees). The $\delta$ 's expected for $\lambda=476.5$ and $568.4 \mathrm{~nm}$ from the values at $514.5 \mathrm{~nm}$ are given in brackets, below the experimental values.

\begin{tabular}{ccccccc}
\hline \hline$\lambda(\mathrm{nm})$ & $\delta(001)$ & $\delta(111)$ & $\delta\left(11 \frac{1}{2}\right)$ & $b / a\left(A^{1}\right)$ & $b / a\left(A^{2}\right)$ & $b / a\left(A^{3}\right)$ \\
\hline & & & 1 & 1 & 0.65 \\
514.5 & 0 & 45 & 48 & & \\
476.5 & 0 & 50 & & & \\
568.4 & 0 & $(48.5)$ & 41.5 & \\
& & $(43.4)$ & \\
\hline \hline
\end{tabular}

reflection coefficients, and assume the same value for the two waves. These parameters would appear as prefactors in Eq. (1). Since we are comparing only relative intensities, and assuming that these factors are isotropic, we can neglect them.

To account for the phase difference we decompose the polarization vectors of the incident and scattered light $\mathbf{e}_{\mathrm{i}}, \mathbf{e}_{\mathrm{s}}$ into a sum of a component perpendicular to the optic axis, which will be the electric field of the ordinary wave, and that of the extraordinary wave, which is the remaining. Then, we will introduce a phase to the ordinary component by multiplying it by a factor $e^{-i \delta}$. With these considerations in mind we can calculate by means of Eq. (1) an expression for the Raman intensity of the nonpolar modes as a function of the polarization direction of the incident light within each plane. We consider the general case as represented in Fig. 1 of light incident along a direction $\mathbf{k} \|(\sin \theta, \sin \theta, \sqrt{2} \cos \theta)$ normal to a plane of cubic indices $(11 l)$, with $l \propto \cot \theta$. All such planes intersect along the $[-110]$ direction so that (001), (111), and $\left(11 \frac{1}{2}\right)$ planes can be obtained as particular cases of the general case. Within such a plane we take reference axes along the directions of polarization of the ordinary and extraordinary waves, with unit vectors given by

$$
\begin{gathered}
\mathbf{r}_{\mathrm{o}}=(1 / \sqrt{2})(-1,1,0), \\
\mathbf{r}_{\mathrm{e}}=(1 / \sqrt{2})(-\cos \theta,-\cos \theta, \sqrt{2} \sin \theta) .
\end{gathered}
$$

If the polarization direction of the incident light is at an angle $\alpha$ from the $[-110]$ direction,

$$
\mathbf{e}_{\mathrm{i}}=\cos \alpha \mathbf{r}_{\mathrm{o}}+\sin \alpha \mathbf{r}_{\mathrm{e}} .
$$

The phase difference between the ordinary and the extraordinary components as the light travels through the medium is introduced in exponential form

$$
\mathbf{e}_{\mathrm{i}^{\prime}}=\cos \alpha e^{-i \delta} \mathbf{r}_{\mathrm{o}}+\sin \alpha \mathbf{r}_{\mathrm{e}} .
$$

The polarization of the scattered light, just after the scattering event, is given by

$$
\mathbf{e}_{\mathrm{s}^{\prime}}=R \mathbf{e}_{\mathrm{i}^{\prime}},
$$

$R$ being the Raman tensor for each $A$ mode. We now decompose $\mathbf{e}_{\mathrm{s}^{\prime}}$, as before, into its ordinary and extraordinary components, as

$$
\mathbf{e}_{\mathrm{s}^{\prime}}=\cos \beta \mathbf{r}_{\mathrm{o}}+\sin \beta \mathbf{r}_{\mathrm{e}}
$$

( $\beta$ being a function of $\alpha, \delta, a$, and $b$ ) and introduce again an $e^{-i \delta}$ factor to account for dephasing as the light travels back toward the surface. On leaving the sample

$$
\mathbf{e}_{\mathrm{s}}=\cos \beta e^{-i \delta} \mathbf{r}_{\mathrm{o}}+\sin \beta \mathbf{r}_{\mathrm{e}} .
$$

Intensities measured in parallel or in crossed configurations can be found simply by projecting $\mathbf{e}_{\mathrm{s}}$ onto either the incident light polarization vector $\mathbf{e}_{i}$, or onto a direction perpendicular to $\mathbf{e}_{\mathrm{i}}$, given by $\mathbf{e}_{\perp}=-\sin \alpha \mathbf{r}_{\mathrm{o}}+\cos \alpha \mathbf{r}_{\mathrm{e}}$, respectively. Then

$$
I_{\|}=\left|\mathbf{e}_{\mathrm{i}} \mathbf{e}_{\mathrm{s}}\right|^{2}, \quad I_{\perp}=\left|\mathbf{e}_{\perp} \mathbf{e}_{\mathrm{s}}\right|^{2} .
$$

More explicitly, $I_{\|}$and $I_{\perp}$ are given by

$$
\begin{aligned}
I_{\|}= & a^{2} \cos ^{4} \alpha+\left(a \cos ^{2} \theta+b \sin ^{2} \theta\right)^{2} \sin ^{4} \alpha \\
+ & 2 a\left(a \cos ^{2} \theta+b \sin ^{2} \theta\right) \sin ^{2} \alpha \cos ^{2} \alpha \cos 2 \delta, \\
I_{\perp}= & \sin ^{2} \alpha \cos ^{2} \alpha\left[a^{2}+\left(a \cos ^{2} \theta+b \sin ^{2} \theta\right)^{2}\right. \\
& \left.-2 a\left(a \cos ^{2} \theta+b \sin ^{2} \theta\right) \cos 2 \delta\right] .
\end{aligned}
$$

The phase difference effect is contained in the last term of each equation. If we make $\delta=0$, Eqs. (2) are reproduced. Since $n_{e f f}=n_{o}$ for the (001) plane, the condition $\delta=0$ holds exactly. Therefore, no dephasing effects should occur in this plane, as observed. For (111) and $\left(11 \frac{1}{2}\right)$ planes we can try to reproduce the experimental angular dependencies shown in Figs. 3 and 4 by means of Eqs. (5). As free parameters we have the tensor components of each mode and the phase difference $\delta$. The parameter relation $a / b$ has been determined from the intensities at $\alpha=0$ and $90^{\circ}$, where no dephasing effects occur. We have therefore only one fitting parameter $(\delta)$ for each plane. It must be noted that $\delta$ depends only on the value of $\theta$, but not on the particular mode, so that a unique $\delta$ value must fit the dependencies of all three $A$ modes within each plane. As seen in Figs. 3 and 4, the reproducibility of the experimental dependencies $I(\alpha)$, as well as the intensity relation $I_{\|}(\alpha) / I_{\perp}(\alpha)$, are quite good, in spite of the approximations made. The parameters which best reproduce the experimental results are given in Table I.

To test the reliability of our model we have performed rotational measurements for other wavelengths. Figure 5 shows the spectra taken in parallel configuration at $\alpha=0$ and $45^{\circ}$ with $\lambda_{1}=476.5 \mathrm{~nm}$ [in the (111) plane $]$ and $\lambda_{2}$ 


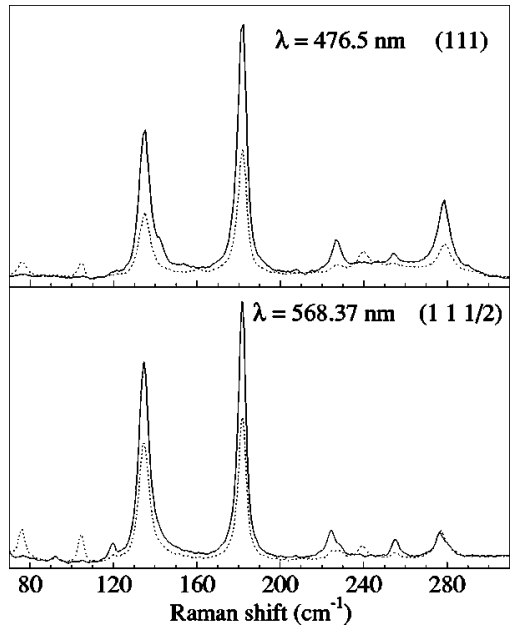

FIG. 5. Raman spectra of $\mathrm{MnGa}_{2} \mathrm{Se}_{4}$ taken with $\lambda_{1}=476.5 \mathrm{~nm}$ (upper graph) and $\lambda_{2}=568.4 \mathrm{~nm}$ (lower graph) in parallel configuration at $\alpha=0$ (continuous line) and $45^{\circ}$ (dashed line).

$=568.4 \mathrm{~nm}$ [in the $\left(11 \frac{1}{2}\right)$ plane]. According to Eq. (4), a higher value of $\delta$ should be found for $\lambda_{1}$ and inversely for $\lambda_{2}$, provided that both the birefringence and the optical path are not strongly dispersive in that narrow region. From the relative intensities of the spectra shown in Fig. 5 we obtain $\delta(476.5 \mathrm{~nm}) \approx 50^{\circ}$ and $\delta(568.4 \mathrm{~nm}) \approx 41.5^{\circ}$ which compare fairly well with the values expected from those at $514.5 \mathrm{~nm}$ through the relation $\delta \propto 1 / \lambda, 48.5^{\circ}$ and $43.4^{\circ}$ for 476.5 and $568.4 \mathrm{~nm}$, respectively.

From these results it derives that the presence of a minimum in $I_{\|}$for the three $A$ modes and the whole intensity $I_{\perp}$ for $A^{1}$ and $A^{2}$ are a consequence of the uniaxial character of the material. Neglecting this fact can yield an incorrect assignment of mode symmetries and tensor elements. Aniso- tropy effects, depending on the parameter values, can either enhance or reduce the angular variations of Raman intensities.

Another interesting result derives also from our calculations: a qualitative estimate of the absolute value of the birefringence can be obtained from the fitting value of $\delta$ and Eq. (4) provided that the optical path is known. [Since $\delta$ appears through a $\cos 2 \delta$ term in Eqs. (5) its sign cannot be determined]. As an example, with $\lambda=514.5 \mathrm{~nm}$ and for the (111) plane we have obtained $\delta$ (in radians) $=\pi / 4$, from where $\Delta d($ in $\mu)=0.0643$. Taking for instance $d=10 \mu$ we would obtain $\Delta=0.00643$.

\section{SUMMARY AND CONCLUSIONS}

The effect of optical anisotropy on the Raman selection rules in uniaxial media has been calculated and applied to nonpolar modes of $\mathrm{MnGa}_{2} \mathrm{Se}_{4}$. Dephasing as high as $50^{\circ}$ have been obtained. Two conclusions can be formulated from our work. The first one is of basic interest and stresses the importance of taking into account the optical anisotropy for the interpretation of Raman spectra in uniaxial media, even if the birefringence is small. Otherwise, erroneous results concerning mode symmetries and tensor elements can derive. The second one is of more practical interest: optical birefringence can be obtained from Raman measurements provided that the optical path run by the light is known. This may be specially interesting for thin films if an accurate control of film thickness is reached.

\section{ACKNOWLEDGMENT}

We acknowledge financial support from research project MAT2001-3713-C04.
*Email address: sanjuan@unizar.es

${ }^{1}$ A. Miller, A. MacKinnon, and D. Weaire, Solid State Phys. 36, 119 (1981).

${ }^{2}$ A. N. Georgobiani, S. I. Radautsan, and I. M. Tiginyanu, Sov. Phys. Semicond. 19, 121 (1985).

${ }^{3}$ L. K. Samanta, D. K. Ghosh, and P. S. Ghosh, Phys. Rev. B 39, 10261 (1989).

${ }^{4}$ V. Petrov and F. Rotermund, Opt. Lett. 27, 1705 (2002).

${ }^{5}$ M. C. Ohmer, R. Pandey, and N. H. Baraimov, MRS Bull. 23, 16 (1998).

${ }^{6}$ A. Anedda, Appl. Opt. 13, 1595 (1974).

${ }^{7}$ L. M. Suslikov, Z. P. Gadmashi, D. S. Kovach, and V. Y. Slivka, Opt. Spektrosk. 53, 480 (1982) [Opt. Spectrosc. 53, 283 (1982].

${ }^{8}$ L. M. Suslikov, Z. P. Gadmashi, M. Y. Rigan, S. I. Perechinsky, and V. Y. Slivka, Opt. Spektrosk. 63, 93 (1987).

${ }^{9}$ J.-D. Hecht, A. Eifler, V. Riede, M. Schubert, G. Frauss, and V. Kramer, Phys. Rev. B 57, 7037 (1998).

${ }^{10}$ M. Cannas, L. Garbato, A. G. Lehmann, N. Lampis, and F. Ledda, Cryst. Res. Technol. 33, 417 (1998).

${ }^{11}$ J. L. Shay and J. H. Wernick, Ternary Chalcopyrite Semiconductors: Growth, Electronic Properties, and Applications
(Pergamon, 1975)

${ }^{12}$ M. C. Morón and S. Hull, Phys. Rev. B 64, 220402(R) (2001).

${ }^{13}$ M. L. Sanjuán and M. C. Morón, Physica B 316-317C, 565 (2002).

${ }^{14}$ A. Gozar, Phys. Rev. B 65, 176403 (2002).

${ }^{15}$ S. P. S. Porto, J. A. Giordmaine, and T. C. Damen, Phys. Rev. 147, 608 (1966).

${ }^{16}$ M. C. Morón and S. Hull, Phys. Rev. B 67, 125208 (2003).

${ }^{17}$ F. Leccabue, B. E. Watts, C. Pelosi, D. Fiorani, A. M. Testa, A. Pajaczkowska, G. Bocelli, and G. Calestani, J. Cryst. Growth 128, 859 (1992).

${ }^{18}$ G. Attolini, S. Bini, P. P. Lottici, and C. Razzetti, Cryst. Res. Technol. 27, 685 (1992).

${ }^{19}$ P. P. Lottici and C. Razzetti, Solid State Commun. 46, 681 (1983).

${ }^{20}$ R. Loudon, Adv. Phys. 13, 423 (1964).

${ }^{21}$ M. Born and E. Wolf, Principles of Optics (Pergamon Press, 1975).

${ }^{22}$ J. P. Mathieu, Optique (Société, d'Édition d'Enseignement Supérieur, 1963). 